\title{
Hierarchical macro-microporous ZIF-8 nanostructures as efficient nano-lipase carriers for rapid and direct electrochemical detection of nitrogenous diphenyl ether pesticides
}

\begin{abstract}
Free biocatalyst such as lipase cannot be recovered and reused; and is extremely unstable in harsh reaction condition. Present study aimed to synthesize hierarchical ordered macro-microporous ZIF-8 structures (MAC-ZIF-8) for encapsulation of nano-Burkholderia cepacia lipase (nano BCL). The synthesized MAC-ZIF-8 has good crystallinity, thermal stability and high surface area which made it suitable for immobilization of nano-BCL. The MAC-ZIF-8 immobilized nano-BCL (nanoBCL@MAC-ZIF-8) demonstrated high enzyme activity (4.3778 U) and can be recovered and reused for consecutive hydrolysis reaction. It is found that the nano-BCL is well encapsulated with the macropores of the MAC-ZIF-8 structures and they are able to preserve their native configuration with high affinity for substrates (lower $\mathrm{Km}$ of $1.068 \mathrm{mM}$ as compared to that of nano-BCL@ZIF-8). The nano-BCL@MAC-ZIF-8 can also be used to fabricate electrochemical biosensors for detection of nitrogenous diphenyl ether pesticides (nitrofen) in real food systems (apricots). The fabricated biosensors demonstrated wide linear range $(0-114 \mu \mathrm{M})$, low limit of detection $(0.46 \mu \mathrm{M})$ and good recovery rate. In comparison to conventional detection method, the fabricated biosensor also has added advantages in the form of easy and convenient operation, low cost, and better linear range.
\end{abstract}

Keyword: Burkholderia cepacia lipase; Lipase nanogelZIF-8; Immobilization; Electrochemical detection; Nitrogenous diphenyl ether pesticides 